\title{
A simple coupling of ALE domain with empirical blast load function in LS-DYNA
}

\author{
L. Gilson ${ }^{1}$, J. Van Roey ${ }^{2}$, C. Guéders² ${ }^{2}$ J. Gallant ${ }^{2}$, and L. Rabet ${ }^{1}$ \\ 1 Royal Military Academy, Department of Civil and Material Engineering, 30 Av. de la Renaissance, \\ 1000 Brussels, Belgium \\ 2 Royal Military Academy, Department of Weapon Systems and Ballistics, $30 \mathrm{Av}$. de la Renaissance, \\ 1000 Brussels, Brussels, Belgium
}

\begin{abstract}
Blast effects on structures are of great interest both in the civilian and military domain. This fact leads to the need of the development of simple and efficient simulation methods. The two common and most used methods have a very different philosophy. The first one is the construction of a full Eulerian domain containing both the detonating charge and the sollicitated structure. An inconvenience of this method is the very long duration of the simulation since the entire external domain is modelled, especially if the charge-structure distance is very large. The second method is the direct application of the corresponding loading based on empirical data (from ConWep) to the structure without simulating the external domain. Although this method allows a consequent time benefit, it has also some disadvantages such as the fact that shadowing, ground reflection and side effects are not taken into account. Moreover, every structure is considered as rigid since the load directly applied is the reflected pressure from ConWep's database, which gives only the reflected pressure in a rigid case. This can be a problem for the application of blast loading on elastic, low-impedance materials such as granular materials or even on small density structures that are able to have a non-negligible displacement during the positive phase duration of the loading. Material impedance and structure displacement might alter the shockwave-structure interaction. A new method using both philosophies has been developed by Slavik [2]. In this method, an Arbitrary Lagrangian-Eulerian mesh (ALE) is created only around the structure in which the empirical incident pressure is applied. The loading is applied onto the air domain, propagates to the structure and interacts with this structure. At this time, the material parameters of the structure and its displacement are taken into account. This paper aims to evaluate third method's capabilities. Moreover, it studies the influence of structure parameters on the loading by a comparison of reflected pressure and impulse.
\end{abstract}

\section{Introduction}

LS-DYNA philosophy is based on parameter cards which, all put together, allow the definition of an entire model. Each of these cards defines a model's characteristic such as geometry, mesh, material model, material parameters, contacts, boundaries ... [1].

The presented application is developed in three steps. The first one is the construction of a quarter symmetric air model composed by two layers on which a blast wave, based on a ConWep empirical function, is applied and propagates through the ALE domain [2,3]. This blast empirical function is defined by the *LOAD_BLAST ENHANCED card.

The second step is the addition of an aluminium plate in the air domain in order to study the Fluid-Structure Interaction (FSI) and the influence of the material parameters on the pressure and impulse.

The third step is the replacement of the elastic material model of the plate by a crushable foam material model with the same purpose as in the second step.

Results are presented such that the user can compare efficiently the different case outcomes. In these results, important considerations about model construction and the influence of material parameters on shockwave-structure interactions are shown.

\section{Blast loading and material parameters}

The application of ConWep's pressure to an Arbitrary Lagrangian-Eulerian mesh requires the creation of two different parts: a thin layer called the ambient layer (AL) composed of a one air element thickness and a thicker layer, the air domain (AD), which can eventually contain other structures. This configuration is represented on figure 1 .

The *LOAD_BLAST_ENHANCED function allows to apply ConWep's empirical static pressure of the incident wave directly on the top of the ambient air layer before it propagates into the air domain.

The ambient layer and air domain are modeled as an ideal gas with a linear polynomial $\gamma$ law equation of state (EOS)

\section{Post-processing}

Figure 2 shows the results of pressure contours on the quarter symmetric model after application of ConWep's pressure on the ambient layer and propagation.

A column of elements at the center of the model going through the entire thickness is selected for pressure analysis (Figure 3). 


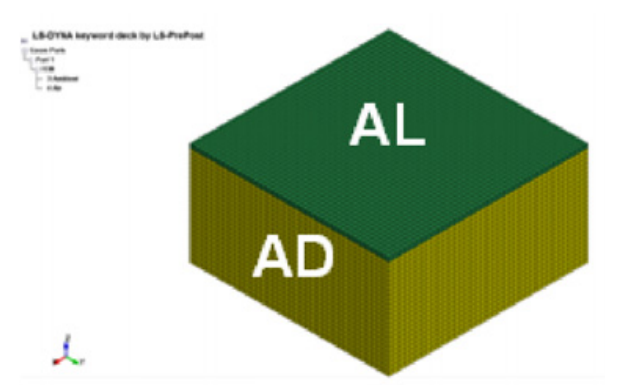

Fig. 1. Model for LOAD_BLAST_ENHANCED application. Uper part (AL) is the ambient layer on which the ConWep function is applied and the lower part (AD) is the air domain.
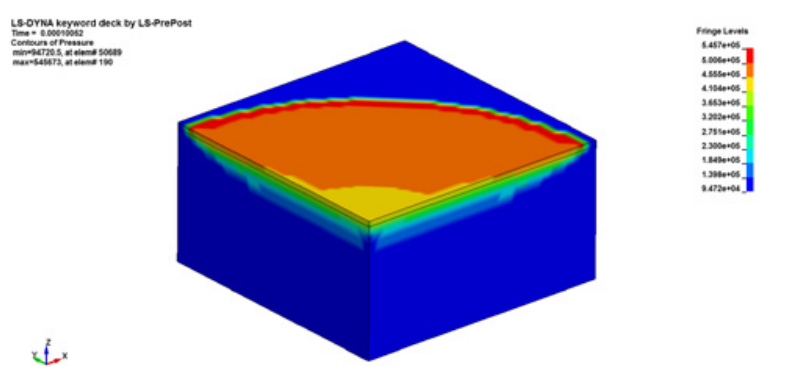

Fig. 2. Pressure visualisation with D3plot.

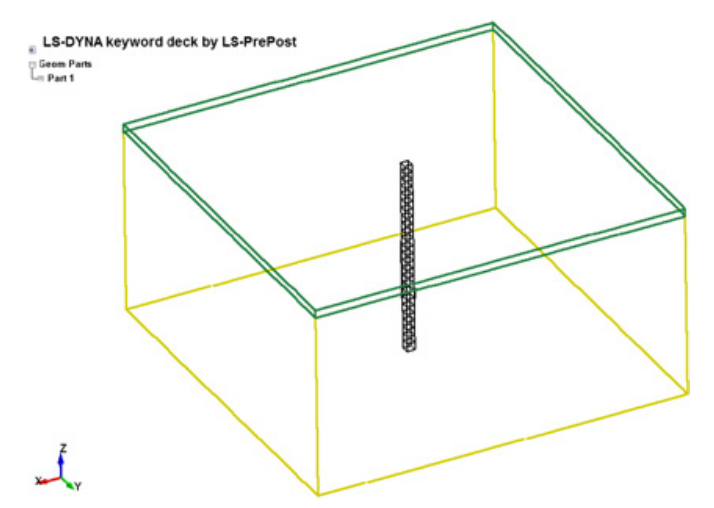

Fig. 3. Elout application to a column of elements.

The Figure 4 shows a graph of the pressure in function of time for the selected column of elements. The first pressure profile represents the pressure in the ambient layer while the following pressure profiles represent pressure in the air domain from the highest layer to the lowest one. The positive phase duration of the air domain profiles is much smaller than the positive phase duration of the ambient layer profile.

The pressure applied in the ambient layer can be compared to ConWep pressure.

Figure 5 shows that this pressure is very close to the empirical blast loading from ConWep.

\section{Plate in the air}

An aluminium plate with a thickness of $0.5 \mathrm{~cm}$ is placed in the air domain $2.5 \mathrm{~cm}$ below the ambient layer-air domain interface (Figure 6). This allows studying fluid-structure

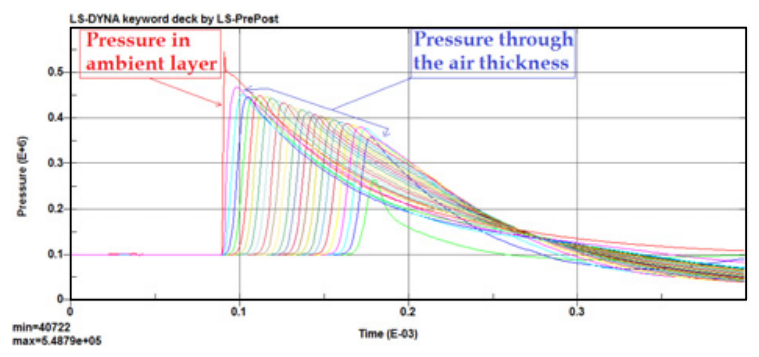

Fig. 4. Pressure profile in the ambient layer and air.

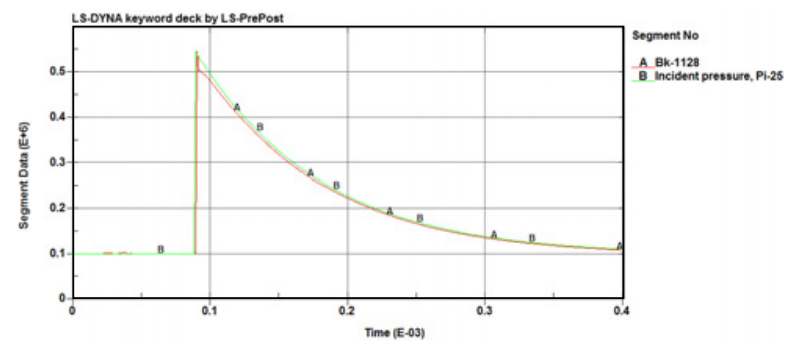

Fig. 5. Comparison between ConWep pressure and LOAD_BLAST_ENHANCED pressure applied to the ambient layer.
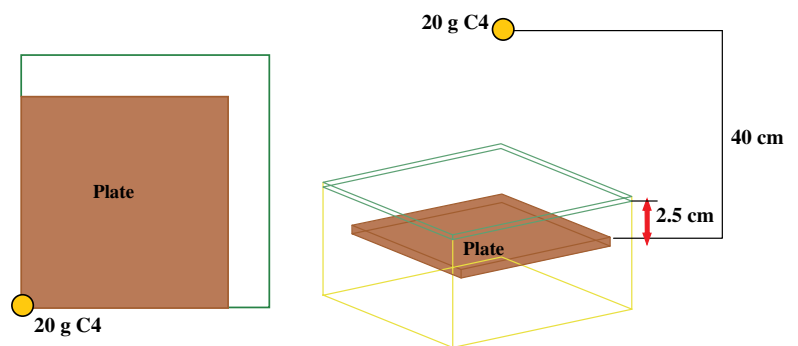

Fig. 6. Plate disposition.

interaction between the plate and shockwaves by comparison of the reflected pressure from the *LOAD_BLAST ENHANCED function and the reflected pressure obtained by this FSI-model. The reflected pressure is measured by the air pressure in the closest cell of the plate surface. The plate material is defined by an elastic material model. The interaction between the aluminum plate and the air domain is defined with the *CONSTRAINED_LAGRANGE_IN SOLID function. The aluminium plate is constrained in all degrees of freedom and can be considered as rigid.

\subsection{Results}

Figure 7 represents the three cells for which pressure curves are displayed on Figure 8. Four curves are presented on Figure 8. Curve $\mathrm{A}$ is the incident pressure in the ambient layer while curve $\mathrm{C}$ is the incident pressure in the first cell of the air domain. Curve $\mathrm{C}$ is clearly in good agreement with the ambient layer cell profile. Curve D is the reflected pressure obtained with ConWep) and curve $\mathrm{B}$ is the pressure in the cell in the air domain just above the plate. The two reflected pressure curves are also very similar, although the curve B is less abrupt and smoothed because of the mesh influence. Moreover, a second and a 


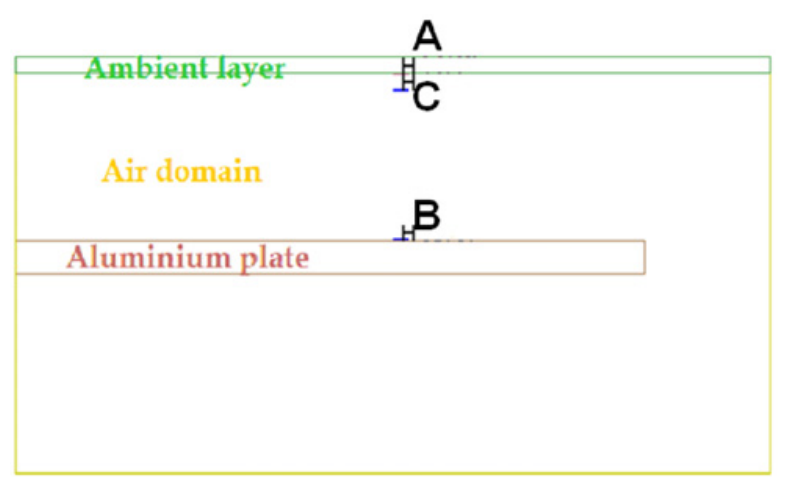

Fig. 7. Reflected pressure in the two air domain cells above the moving elastic plate.

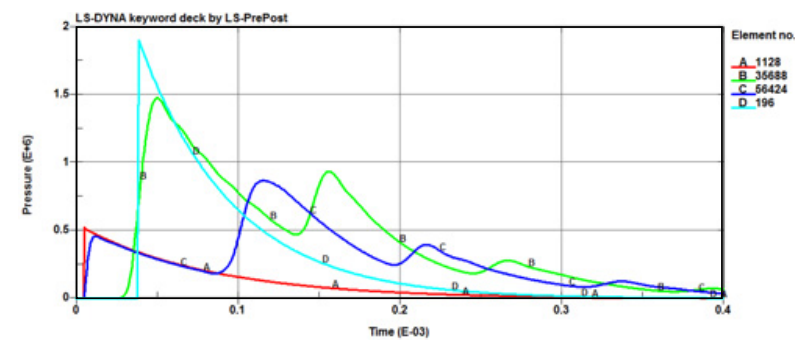

Fig. 8. Incident and reflected pressure for an interface-plate distance of $2.5 \mathrm{~cm}$.

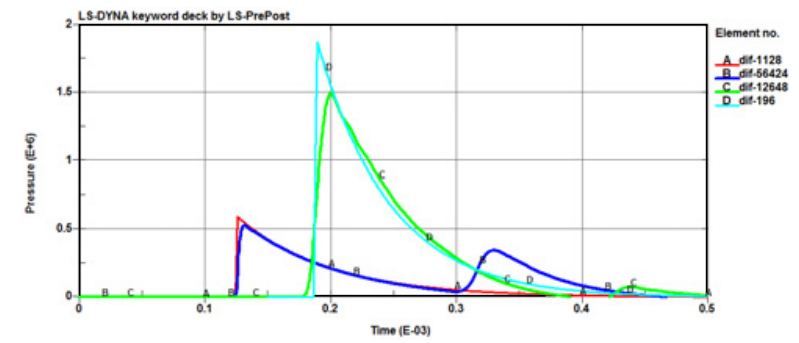

Fig. 9. Incident and reflected pressure for a plate-interface distance of $5 \mathrm{~cm}$.

third pressure peaks are visible on both FSI-curves; these effects are due to shockwave reflection on the border of the air domain.

First, incident pressure is applied to the ambient layer and goes through the air domain until the reflection on the plate, giving the reflected pressure. Secondly, the shockwave goes back through the air domain and reflects on the ambient layer-air domain interface, creating the peak visible at about $0.11 \mathrm{~ms}$ on curve $\mathrm{C}$. Then, the shockwave goes through the air domain again and reflects on the plate, creating the second peak on curve B, and so on. Shock wave velocity can be evaluated by the distance divided by the time taken by the shockwave to travel this distance and is about $720 \mathrm{~m} / \mathrm{s}$ in this case.

A second simulation was run with an interface-plate distance of $5 \mathrm{~cm}$ instead of the $2.5 \mathrm{~cm}$ initially set. Pressure results are shown on figure 9 and impulse results are shown on figure 10 .

Pressure profiles obtained with a higher distance between the interface and the plate avoid reflection peaks during the application of the reflected pressure (curve C).

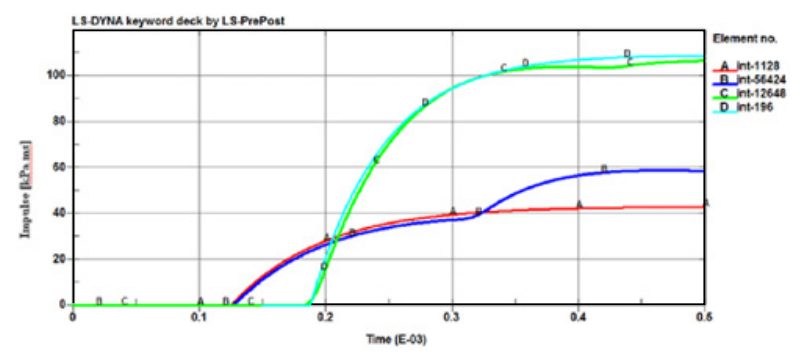

Fig. 10. Incident and reflected impulse for a plate-interface distance of $5 \mathrm{~cm}$.

Table 1. Comparison of reflected overpressure and impulse of cases 1,2 and 3 .

\begin{tabular}{|c|c|c|c|}
\hline Conditions & $\begin{array}{c}\text { Max. } \\
\text { overpressure } \\
{[\mathbf{k P a}]}\end{array}$ & $\begin{array}{c}\text { Max. } \\
\text { impulse } \\
{[\mathbf{k P a} . m s]}\end{array}$ \\
\hline 1 & $\begin{array}{c}\text { Reference (rigid) } \\
\text { Crushable Foam - lower }\end{array}$ & 1498 & 104.01 \\
\hline 2 & $\begin{array}{c}\text { surface blocked Crushmat } \\
\text { data } \\
\text { Crushable Foam - lower }\end{array}$ & 1435 & 101.38 \\
\hline 3 & $\begin{array}{c}\text { surface blocked } \\
\text { Vermiculite data }\end{array}$ & 1237 & 99.40 \\
\hline
\end{tabular}

This is due to the distance between the plate and the interface which is now high enough in order that the shockwave front has not the time to go back through the air domain and come back again. Impulse becomes now a worthy result to show; very good agreements are found between ConWep's and FSI profiles, although the distance interface-plate is not yet enough for avoiding reflection in the incident pressure profile (curve B).

\section{Crushable foam applied as plate material model}

Replacing the elastic plate material model by a crushable foam model can be interesting since this model could be used for granular or soft materials modeling encountered in shock absorbing applications. In this part, two different granular materials are modeled: CRUSHMATßand vermiculite. The lower surface of the plate remains blocked.

\subsection{Crushable foam material model definition}

Crushmat is modeled as a homogenous material with a density of $600 \mathrm{~kg} / \mathrm{m}^{3}$, an elastic modulus $E=68.97 \mathrm{GPa}$

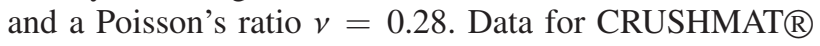
material come from Niras-Demex [4].

Vermiculite is modeled as a homogenous material with a density of $140 \mathrm{~kg} / \mathrm{m}^{3}$. The elastic modulus E and Poisson's ratio $v$ are not known and set to 0 .

Stress - strain curves are obtained from confined static uniaxial compression tests (oedometric tests). 


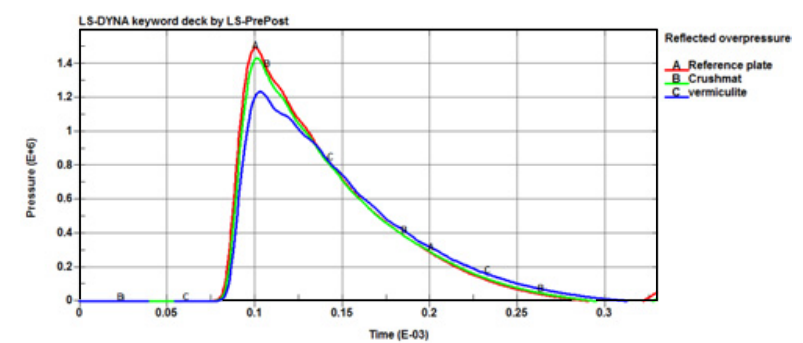

Fig. 11. Comparison of reflected overpressure of cases 1, 2 and 3.

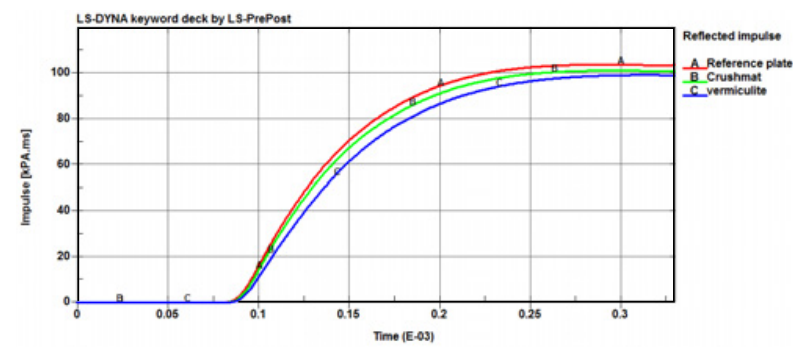

Fig. 12. Comparison of reflected impulse of cases 1, 2 and 3.

\section{Results}

The elastic material of the plate is replaced by a crushable foam model. The above surface of the plate is blocked in all degree of freedom. The results of the different simulations are given in table 1 . Figure 11 and 12 respectively represent the reflected pressure and impulse in the air cell just above the plate.

Reflected pressure values are influenced by the material parameters. Pressure decrease is about $5 \%$ for CRUSHMAT and about $20 \%$ for vermiculite.
On the contrary, reflected impulse remains relatively constant.

\section{Conclusion}

The capabilities of coupling empirical blast loading to an ALE domain have been demonstrated although some elements have to be taken into account. The most important is certainly the distance between the ambient layer-air domain interface and the plate that has to be high enough in order to cancel reflection effects. Moreover, a small enough mesh size is also important to avoid too much smoothed peak pressure.

Material parameters of the structure seem to have a non-negligible influence on maximum pressure.

\section{References}

1. LS-DYNA Keyword User's Manual Version 971, Livermore Software Technology Corporation, Livermore, (2007)

2. T.P. Slavik, A Coupling of Empirical Explosive Blast Loads to ALE Air Domains in LS-DYNA, Livermore Software Technology Corporation. $7^{\text {th }}$ European LSDYNA Conference

3. L. Schwer. A Brief Introduction to Coupling Load Blast Enhanced with Multi-Material ALE: The Best of Both Worlds for Air Blast Simulation. LS-DYNA Forum, Bamberg, (2010)

4. M.B. Larsen and K.C. Jorgensen. Landmine Protection of Armoured Personnel Carrier. $6^{\text {th }}$ European LSDYNA Users' Conference, Gothenburg, (2007) 